\title{
ON SOLUTIONS OF NTH ORDER LINEAR DIFFERENTIAL EQUATIONS WITH $N$ ZEROS
}

BY THOMAS L. SHERMAN ${ }^{1}$

Communicated by Wolfgang Wasow, March 22, 1968

In a very interesting paper in 1958, Hartmann proved the following result [2]: The equation

$$
L_{n} y=y^{(n)}+p_{n-1}(x) y^{(n-1)}+\cdots+p_{0} y=0,
$$

with $p_{i} \in C(a, b)$, has a nontrivial solution with $n$ zeros, each zero being counted in accordance with its multiplicity, if and only if there is a solution with $n$ distinct zeros on $(a, b)$.

Left as an open question in that paper was whether the result remains true when the restriction that the interval be open is removed. The answer to that question is yes if the interval is half-open and no if it is closed. In addition, there is the problem of giving a more exact description to the solution with distinct zeros, that is, does there exist a solution with exactly $n$ distinct zeros all of which are simple? The answer to this question is also yes.

As an application of these results it is established that the first conjugate point $\eta_{1}(t)$ of the point $t$ is a continuous function of the coefficients in (1). It is, by the way, not difficult using the techniques in [3] to establish that $\eta_{1}(t)$ is a continuous function of $t$; however, an easier proof is available using the results stated herein. The results of this paper are stated in terms of equation (1) but could just as easily be stated for a more general equation as in [4].

Results. By a solution it is understood that what is meant is a nontrivial solution.

DeFinition 1. Let $\eta_{1}(a)$, the first conjugate point of $a$, be the smallest $b>a$ such that there is a solution of (1) with $n$ zeros on $[a, b]$.

THEOREM 1. The equation (1) has a solution with $n$ zeros on $[a, b)$ if and only if there is a solution of (1) with a simple zero at a, whose first $n$ zeros on $[a, b)$ are simple zeros.

In order to establish this result a number of lemmas are required some of which are of interest in their own right. The most important of these lemmas is stated below.

1 This work was supported by the National Science Foundation under Grant \#GP-7398. 
LEMMA 1. Suppose there is a solution $\phi(x)$ of (1) with $n$ zeros on $[a, b)$, $a$ zero at $a$, and $R-1$ distinct zeros on $[a, \eta,(a))$. Suppose also no solution of (1) with a zero at $a$ and $n$ zeros on $[a, b)$ has more than $R-1$ distinct zeros on $\left[a, \eta_{1}(a)\right)$. Then the zeros of $\phi$ in $\left(a, \eta_{1}(a)\right)$ are simple zeros.

THEOREM 2. The following are equivalent:

(i) There is a solution of (1) with $n$ zeros on $(a, b)$.

(ii) There is a solution of (1) with $n$ zeros on $[a, b)$.

(iii) There is a largest point $\alpha \in(a, b)$ such that for any $c \in[a, \alpha)$, $\eta_{1}(c)<b$ and for this point $\alpha, \eta_{1}(\alpha) \notin(a, b)$.

(iv) There is a largest point $\alpha \in(a, b)$ such that for any $c \in[a, \alpha)$, there is a solution with a simple zero at $c$ whose first $n$ zeros on $[c, b)$ are simple zeros.

The following example shows that the interval $[a, b)$ cannot in general be closed at $b$. The equation $y^{\prime \prime \prime}+y=0$ has the property that $\eta_{1}(0)$ is achieved by a solution $\phi$ with a double zero at 0 and a simple zero at $\eta_{1}(0)$. Further, no nontrivial solution with a double zero at $\eta_{1}(0)$ can vanish in $\left[0, \eta_{1}(0)\right)$. If there were a solution $\Psi$ with three distinct zeros on $\left[0, \eta_{1}(0)\right]$, then it would have to vanish at 0 and have a simple zero at $\eta_{1}(0)$. Hence there would be a solution which is a linear combination of $\phi$ and $\Psi$ with zero at 0 and a double zero at $\eta_{1}(0)$, which is impossible. A further discussion of equations of this type can be found in [1]. The contradiction arrived at in this example is an application of

THEOREM 3. Suppose there is a solution of (1) with $n$ zeros on $\left[a, \eta_{1}(a)\right]$, and at least $\alpha$ zeros in $\left[a, \eta_{1}(a)\right)\left(\left(a, \eta_{1}(a)\right]\right)$. Then there is a solution of (1) with a zero of order at least $\alpha$ at $a\left(\eta_{1}(a)\right)$ and $n$ zeros on $\left[a, \eta_{1}(a)\right]$.

To give a more complete picture of the situation the following results are presented which give an idea of what is true when there is a solution with $n$ distinct zeros on $\left[a, \eta_{1}(a)\right]$. It is easy to show that if there is a solution with $n$ distinct zeros on $\left[a, \eta_{1}(a)\right]$, then these zeros are simple zeros.

THEOREM 4. Suppose there is a solution $\phi(x)$ of (1) with $n$ simple zeros on $\left[a, \eta_{1}(a)\right]$. Then there exist solutions $\phi_{2}, \phi_{3}, \cdots, \phi_{n-1}$ such that $\phi_{k}$ has $n$ zeros on $\left[a, \eta_{1}(a)\right]$, a zero at a of order $k, n-k-1$ simple zeros in $\left(a, \eta_{1}(a)\right)$ and no other zeros in $\left[a, \eta_{1}(a)\right)$. Further, the zeros of $\phi_{k+1}(x)$ in $\left(a, \eta_{1}(a)\right)$ coincide with the first $n-k-2$ zeros of $\phi_{k}(x)$.

THEOREM 5. Suppose there is a solution of (1) with $n$ simple zeros on $\left[a, \eta_{1}(a)\right]$. Then there exists solutions $\Psi_{1}, \Psi_{2}, \cdots, \Psi_{n-1}$, not necessarily 
distinct, such that $\Psi_{k}$ has a zero at a of order at least $n-k$ and a zero at $\eta_{1}(a)$ of order at least $k$.

By way of a partial converse to these results the following theorem is given:

THEOREM 6. Suppose there are $n-1$ linearly independent solutions of (1), $\Psi_{1}, \cdots, \Psi_{n-1}$ such that $\Psi_{k}$ has a zero at a of order exactly $n-k$, a zero at $\eta_{1}(a)$ of order exactly $k$, and $\Psi_{k}$ does not vanish in $\left(a, \eta_{1}(a)\right)$. Then there are $n-1$ solutions $\phi_{1}, \cdots, \phi_{n-1}$ such that $\phi_{k}$ has a zero at a of order exactly $[(n-k+2) / 2]$, a zero at $\eta_{1}(a)$ of order exactly $[(n-k+1) / 2]$ and exactly $n-[(n-k+2) / 2]-[(n-k+1) / 2]$ distinct zeros in $\left(a, \eta_{1}(a)\right)$, where $[x]$ denotes the largest integer not exceeding $x$. In particular, there is a solution, namely $\phi_{n-1}$, with $n$ simple zeros on $\left[a, \eta_{1}(a)\right]$.

The proofs of these results will appear elsewhere.

\section{BIBLIOGRAPHY}

1. M. Hanan, Oscillation criteria for third-order linear differential equations, Pacific J. Math. 11 (1961), 919-944.

2. P. Hartmann, Unrestricted n-parameter families, Rend. Circ. Mat. Palermo 7 (1958), 123-142.

3. T. Sherman, Properties of solutions of Nth order linear differential equations, Pacific J. Math. 15 (1965), 1045-1060.

4. T. Sherman, Properties of solutions of quasi-differential equations, Duke Math. J. 32 (1965), 297-304.

Arizona State University 